\title{
Early response to neoadjuvant chemotherapy can help predict long-term survival in patients with cervical cancer
}

\author{
Xiong Li ${ }^{1,2, *}$, Kecheng Huang ${ }^{1, *}$, Qinghua Zhang ${ }^{1,2, *}$, Jian Shen ${ }^{2}$, Hang Zhou ${ }^{3}$, \\ Runfeng Yang ${ }^{4}$, Lin Wang ${ }^{1}$, Jiong Liü ${ }^{5}$ Jincheng Zhang ${ }^{6}$, Haiying Sun ${ }^{1}$, Yao Jia ${ }^{1}$, \\ Xiaofang Du ${ }^{1,2}$, Haoran Wang ${ }^{7}$, Song Deng ${ }^{8}$, Ting Ding ${ }^{1}$, Jingjing Jiang ${ }^{1}$, Yunping \\ Lu $^{1}$, Shuang Li ${ }^{1}$, Shixuan Wang ${ }^{1}$ and Ding $\mathrm{Ma}^{1}$ \\ ${ }^{1}$ Department of Obstetrics and Gynecology, Tongji Hospital, Tongji Medical College, Huazhong University of Science and \\ Technology, Wuhan, Hubei, China \\ 2 Department of Obstetrics and Gynecology, Wuhan Central Hospital, Wuhan, Hubei, China \\ ${ }^{3}$ Department of Obstetrics and Gynecology, Nanjing Drum Tower Hospital, The Affiliated Hospital of Nanjing University \\ Medical School, Nanjing, Jiangsu, China \\ ${ }^{4}$ Hubei Tumor Hospital, Wuhan, Hubei, China \\ ${ }^{5}$ Shanghai Jiao Tong University School of Medicine, Shanghai, China \\ ${ }^{6}$ Xinhua Hospital, Shanghai Jiao Tong University School of Medicine, Shanghai, China \\ 7 Department of Internal Medicine, Luohe Renmin Hospital, The First Affiliated Hospital of Luohe Medical College, Luohe, \\ Henan, China \\ ${ }^{8}$ Department of Obstetrics and Gynecology, University Hospital of Hubei University for Nationalities, Enshi, Hubei, China \\ * These authors have contributed equally to this work \\ Correspondence to: Shuang Li, email: lee5190008@126.com
}

Shixuan Wang, email: sxwang2012@hotmail.com;

Ding Ma, email: dma@tjh.tjmu.edu.cn

Keywords: cervical cancer, clinical response, neoadjuvant chemotherapy (NACT), predictor, disease-free survival (DFS) Received: April 11,2016 Accepted: August 13, $2016 \quad$ Published: August 20, 2016

\section{ABSTRACT}

It is still controversial whether cervical cancer patients with clinical responses after neoadjuvant chemotherapy (NACT) have a better long-term survival or not. This study was designed to investigate the effect of the clinical response on the disease-free survival (DFS) of cervical cancer patients undergoing NACT. A total of 853 patients from a retrospective study were used to evaluate whether the clinical response was an indicator for the long-term response, and 493 patients from a prospective cohort study were used for further evaluation. The survival difference was detected by log-rank test, univariate and multivariate Cox regression and a pooled analysis. The log-rank test revealed that compared with non-responders, the DFS of responders was significantly higher in the retrospective data $(P=0.007)$. Univariate Cox regression showed that the clinical response was an indicator of longterm survival in the retrospective study (HR 1.83, 95\% CI 1.18-2.85, $P=0.007$ ). In a multivariate Cox model, the clinical response was still retained as an independent significant prognostic factor in the retrospective study (HR 1.59, 95\% CI 1.01-2.50, $P=0.046$ ). The result was also validated in the prospective data with similar results. These findings implied that the clinical response can be regarded as an independent predictor of DFS.

\section{INTRODUCTION}

Cervical cancer is the second common malignant tumor-causing disease in women in undeveloped countries
[1]. East Asia contributes more than $12 \%$ of new global cases or deaths [2,3], and more than 130,000 new patients and 50,000 deaths each year [4]. As demonstrated by the National Comprehensive Cancer Network (NCCN) 
Table 1: Clinical characteristics of all patients.

\begin{tabular}{|c|c|c|c|c|}
\hline \multirow{2}{*}{ Characteristics } & \multicolumn{2}{|c|}{ Retrospective $(n=853)$} & \multicolumn{2}{|c|}{ Prospective $(n=493)$} \\
\hline & No. & $\%$ & No. & $\%$ \\
\hline \multicolumn{5}{|c|}{$\begin{array}{c}\text { Age (25th-75th percentiles) } \\
\text { (year) }\end{array}$} \\
\hline Median & \multicolumn{2}{|c|}{44} & \multicolumn{2}{|c|}{45} \\
\hline Range & \multicolumn{2}{|c|}{$39-50$} & \multicolumn{2}{|c|}{$40-49$} \\
\hline \multicolumn{5}{|c|}{$\begin{array}{c}\text { Tumor size }(25 \text { th-75th } \\
\text { percentiles })(\mathrm{cm})\end{array}$} \\
\hline Median & \multirow{2}{*}{\multicolumn{2}{|c|}{$\begin{array}{l}4.0 \\
3.5-5.0 \\
\end{array}$}} & \multicolumn{2}{|c|}{4.0} \\
\hline Range & & & \multicolumn{2}{|c|}{$3.0-5.0$} \\
\hline \multicolumn{5}{|l|}{ Tumor grade } \\
\hline G1 & 58 & 6.8 & 36 & 7.3 \\
\hline $\mathrm{G} 2$ & 354 & 41.5 & 221 & 44.8 \\
\hline G3 & 240 & 28.1 & 162 & 32.9 \\
\hline Undetermined & 201 & 23.6 & 74 & 15.0 \\
\hline \multicolumn{5}{|l|}{ FIGO stage } \\
\hline IB2 & 220 & 25.8 & 127 & 25.8 \\
\hline IIA & 265 & 31.1 & 117 & 23.7 \\
\hline IIB & 368 & 43.1 & 249 & 50.5 \\
\hline \multicolumn{5}{|l|}{ Cell type } \\
\hline Squamous & 756 & 88.6 & 436 & 88.4 \\
\hline $\begin{array}{l}\text { Non-squamous } \\
\text { Unknown }\end{array}$ & $\begin{array}{c}91 \\
6 \\
\end{array}$ & $\begin{array}{c}10.7 \\
0.7\end{array}$ & $\begin{array}{c}53 \\
4 \\
\end{array}$ & $\begin{array}{c}10.8 \\
0.8\end{array}$ \\
\hline
\end{tabular}

FIGO, International Federation of Gynecology and Obstetrics.

guidelines and the International Federation of Gynecology and Obstetrics (FIGO) system, neoadjuvant chemotherapy (NACT) plus surgery has emerged as a promising therapy for locally advanced cervical cancer. First, NACT can help reduce tumor size, diminish the expansion of tumors [5], and eliminate distant metastasis [6-9]. Consequently, more patients get the chance for surgery, leading to great improvements in their quality of life [10]; both young and old women benefit from NACT plus surgery, as young women can have their fertility preserved [11, 12], pre-menopausal women can have their sexual function preserved, and old women can get avoid the severe side effects of radiation therapy. Meanwhile, previous studies have also demonstrated that long-term survival may be significantly increased by NACT plus surgery compared with surgery alone or radiation therapy $[6,13,14]$.

However, it is still controversial whether cervical cancer patients with clinical responses after NACT have better long-term survival. Many studies have shown that the clinical response is not a significant prognostic predictor or that it may not have an effect on survival [1518], although our previous study revealed that clinical responders had a higher survival rate than non-responders [19].

The sample size of the previous studies was relatively small; thus, bias may exist. This study was designed to test whether the clinical response to NACT was a predictor of long-term survival among patients with cervical cancer in a large retrospective study; meanwhile, data from a prospective cohort were also used for further validation.

\section{RESULTS}

In the retrospective analysis, we included 853 patients with stage IB2-IIB cervical cancer receiving neo-adjuvant platinum-based chemotherapy and radical hysterectomy (Table 1). The median age of the patients at the time of study entry was 44 (range 39-50) years. In the prospective cohort, which was also used in the previous study, 493 patients were included in the beginning, all of whom underwent neo-adjuvant platinum-based chemotherapy and radical hysterectomy (Supplementary Figure 1); the details are listed in Table 1.

\section{Log-rank test}

The log-rank test was used to compare the difference in survival between the responders and non-responders, while the Kaplan-Meier method was used to draw a survival curve. The results revealed that the responders had significantly superior survival to the non-responders $(P=0.007$ for DFS in the retrospective study and $P=$ 0.004 for DFS in the prospective study), and the KaplanMeier plot also showed that the responders had higher 
survival rates than the non-responders.

\section{Univariate Cox regression analysis}

In both the retrospective study and the prospective cohort study, the difference in survival was compared between the responders and non-responders. A Cox proportional hazard regression model was used to evaluate the effect of the risk factors effect on survival; the potential risk factors included clinical response, age, tumor size, FIGO stage, cell type, grade, lymph vascular space invasion (LVSI), parametrial infiltration, vaginal surgical margin and lymph node metastasis. A forest plot was employed to illustrate the HR and its $95 \%$ CI based on univariate Cox regression analysis. In the retrospective study, the clinical response was an indicator of DFS with statistical significance as the responders had superior survival rates with a HR of 1.83 (95\% CI 1.18 to $2.85 ; P$ $=0.007$ ) compared with the non-responders (Figure 2). In the prospective cohort study (Figure 3 ), the clinical response was also a significant indicator of DFS (HR 2.50; $95 \%$ CI 1.44 to $4.34 ; P=0.001)$. More details are listed in Supplementary Table 2 and Supplementary Table 3.

\section{Multivariate Cox regression analysis}

Multivariate Cox proportional hazard regression was used to evaluate whether the clinical response was an independent prognostic factor for long-term survival. With similar methods as those used above, all the risk factors, including clinical-response, were assessed in the retrospective study (Figure 4) and in the prospective cohort (Figure 5). The results showed that compared with the non-responders, the responders had superior survival rates after adjustment with all the risk factors; the factor had a HR of 1.59 for DFS (95\% CI 1.01 to $2.50 ; P=$ $0.046)$ in the retrospective study (Supplementary Table 4) and a HR of 2.09 for DFS (95\% CI 1.10 to $4.00 ; P=0.02$ ) in the prospective cohort (Supplementary Table 5).

\section{Combined results of Cox analysis of the clinical response}

The results from the retrospective study and the prospective study were combined together according to the method illustrated in the previous study [20]. In univariate Cox analysis, the HR was 2.07 (95\% CI, 1.46 to 2.92) (Figure 6A). In multivariate Cox analysis, the HR was 1.74 (95\% CI, 1.20 to 2.53) (Figure 6B).

\section{Survival difference in each FIGO stage}

After the data from the two studies was combined, a log-rank test was used to compare the difference in DFS between the responder and the non-responder groups for each FIGO stage; meanwhile, the Kaplan-Meier method
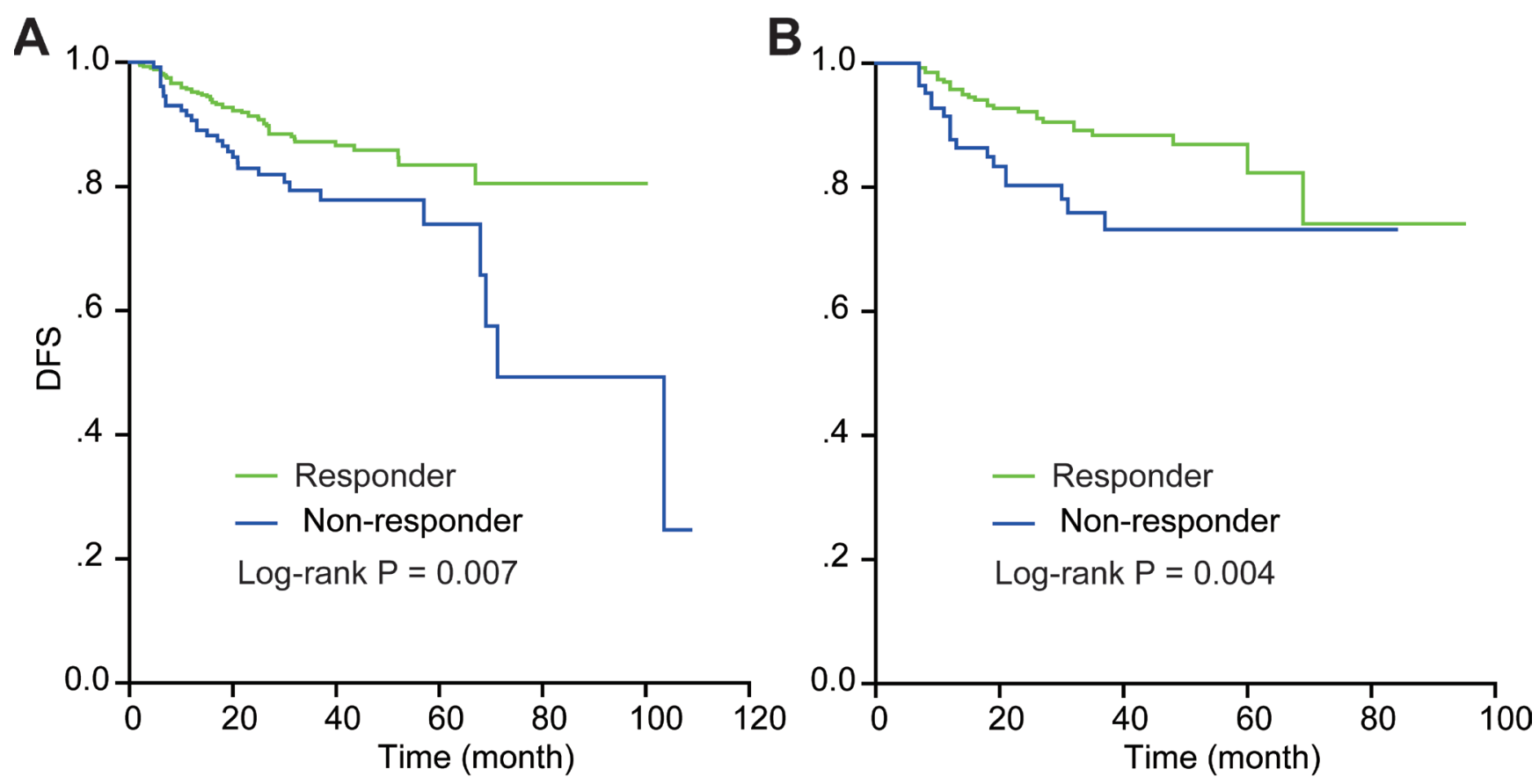

Figure 1: Kaplan-Meier survival estimates for responders and non-responders in the retrospective study and the prospective study. Disease-free survival (DFS) curves of the evaluated patients in the retrospective study A. and the prospective cohort B. are shown. A log-rank test used to calculate the $P$ values. Statistical significances were observed between the responders and the nonresponders. 
was used to draw a survival curve. The results revealed that responders had superior survival to non-responders with statistical significance in each stage $(P<0.05)$ as the Kaplan-Meier plot also showed that the responders had higher disease-free survival rates than non-responders (Figure 7).

High-risk prognostic factors' distribution among responders and non-responders

After the data from the two studies was combined, three high-risk prognostic factors were explored, including vaginal surgical margin, parametrial infiltration and lymph node metastasis (Figure 8). All three high-risk prognostic factors occurred more frequently in the nonresponder group, compared with the responder group (Supplementary Figure 2). The role of chemotherapy on responders' post-surgery treatment was also shown in Supplementary Figure 3.

\section{DISCUSSION}

This study combined data from a large case-control study and a prospective cohort study together to test the effect of the clinical response on long-term survival. In this study, clinical response was demonstrated to be an independent prognostic factor, as responders had significant higher DFS rates than non-responders.

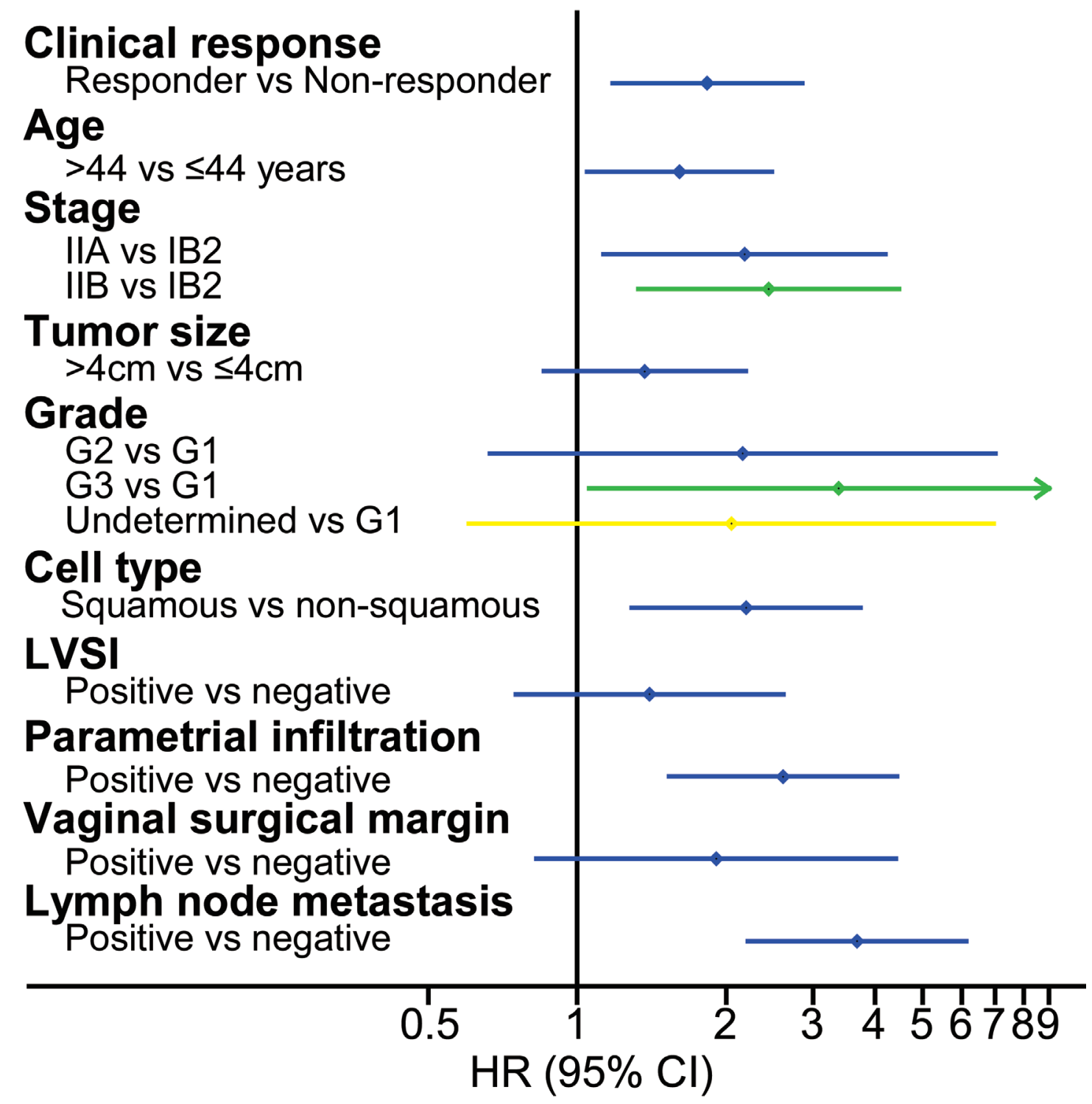

Figure 2: Univariate prognostic factor analysis in the retrospective study. Univariate Cox analysis showed that several factors were significantly associated with DFS, including clinical response, age, FIGO stage, grade (G3 vs G1), cell type, parametrial infiltration and lymph node metastasis. Abbreviations: HR, hazard ratio; FIGO, International Federation of Gynecology and Obstetrics; DFS, diseasefree survival; LVSI, lymph vascular space invasion. 
It was discovered that being a clinical responder was a factor indicating a better DFS in the retrospective phase. This finding was also validated in patients with cervical cancer in a prospective cohort. Meanwhile, as NACT has been used in the treatment of cervical cancer for many years, previous studies have also revealed that short-term response is an indicator of long-term survival. Scholars (Xiong and colleagues) in Sun YatSen University demonstrated that non-responders had relatively lower survival rates than responders [21]; others (Li and colleagues at Chongqing Medical University; Chen and colleagues at Wuhan University) also made the similar discovery $[22,23]$. This study also validated other researchers' findings as well as our previous results [19, 21-24]. Thus, we believe that early clinical response to chemotherapy can be treated as an indicator to DFS.

Our study also investigated a high-risk pathological factor, lymph node metastasis, as well as its relationship with DFS; our finding is consistent with previous findings. $\mathrm{Li}$ and colleagues discovered that patients without lymph node metastasis also achieved significantly longer survival [22], and Biewenga and colleagues demonstrated that lymph node metastasis led to worse overall survival [25]. Sevin and colleagues also revealed its prognostic effect on long-term survival [26]; Kamura and colleagues demonstrated that the presence of positive nodes had a negative effect on long-term survival with statistical significance [27]; and Lai and colleagues discovered that patients with positive nodes also achieved significantly shorter survival periods" [28]. Ho and colleagues also demonstrated that lymph node metastasis was an independent prognostic factor [29]; Alvarez and colleagues also revealed its prognostic effect on long-term survival [30]; Yuan and colleagues demonstrated that positive nodes led to worse overall survival [31]; and Deng and colleagues demonstrated that lymph node metastasis was

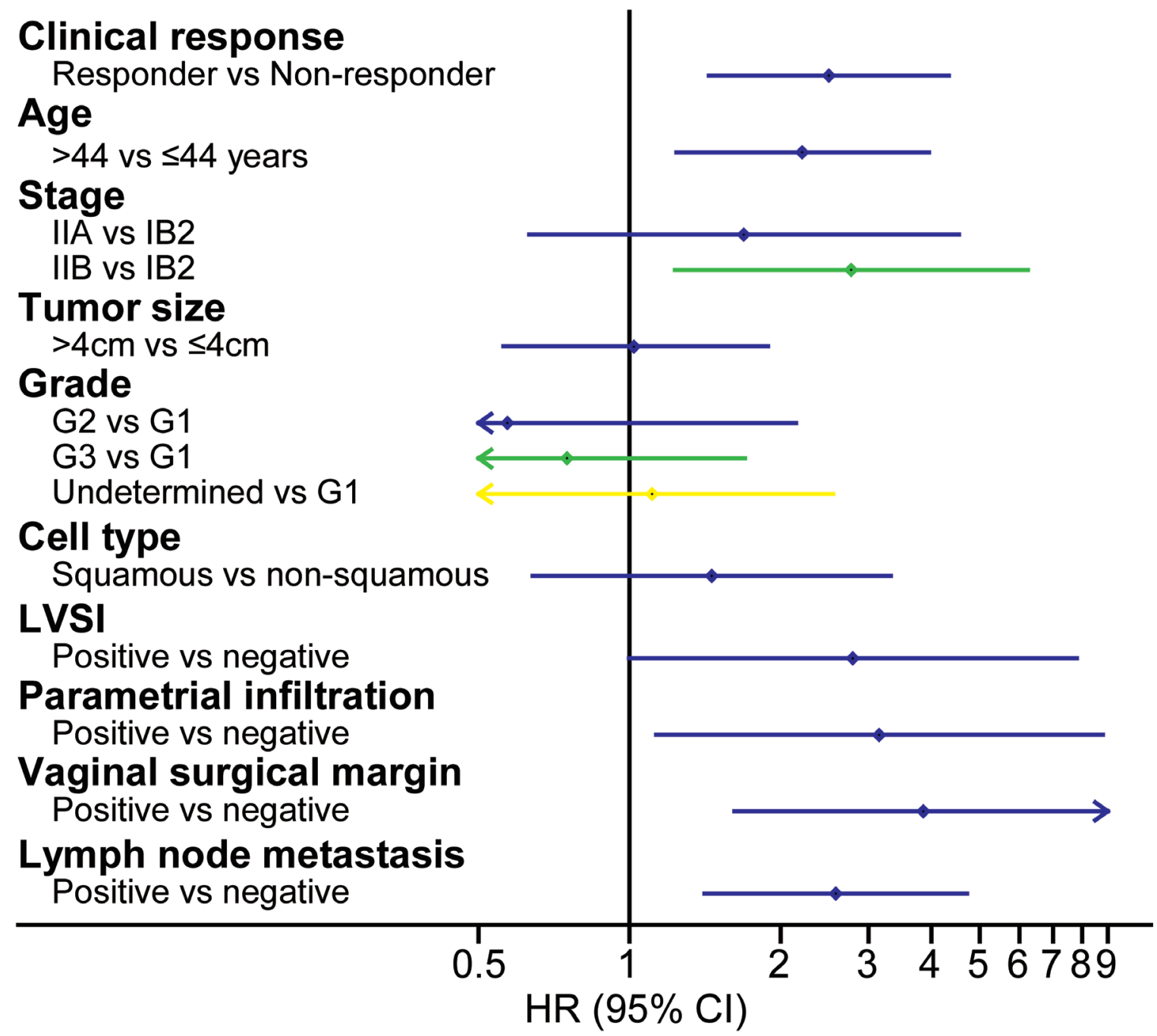

Figure 3: Multivariate prognostic factor analysis in the retrospective study. Multivariate Cox analysis showed that several factors were significantly associated with DFS, including clinical response, FIGO stage, grade (G3 vs G1), cell type, parametrial infiltration and lymph node metastasis. Abbreviations: HR, hazard ratio; FIGO, International Federation of Gynecology and Obstetrics; DFS, diseasefree survival; LVSI, lymph vascular space invasion. 
a significant independent poor prognostic factor after multiple Cox regression analysis [32]. Thus, our research validated the previous findings of other scholars.

Our study also investigated high-risk factors, such as parametrial infiltration and vaginal surgical margin. Compared with responders, the high-risk factors were more frequently observed in the non-responders with statistically significance. We also validated the previous findings made by other scholars; Giaroli and colleagues also demonstrated that parametrial infiltration was an independent prognostic factor [33], as did other scholars $[25,34]$. Furthermore, $\mathrm{Hu}$ and colleagues demonstrated that positive vaginal surgical margin had a negative effect on long-term survival with statistical significance [35], as did Suprasert and colleagues [36].

Although grade, cell type and LVSI were regarded as potential risk factors, our study failed to demonstrate these factors to be independent prognostic factors in multivariate Cox analysis. This is partly because NACT have eliminated the potential risk [19, 37], and thus, patients with G2/G3 differentiation, non-squamous cell type or positive LVSI also had excellent prognoses.
However, our study has some limitations. First, biomarkers that may contribute to the long-term survival of cervical cancer patients have not been investigated by us. Second, the reason why some patients achieved a clinical response but some patients did not has not been explored in this study. Third, a pathological response was also reported to be an important prognostic factor [38]; however, it was not evaluated in this study. In further studies, we will explore the role of biomarkers in survival, the reason why some women can achieve clinical response while others do not, and the role that the pathological response plays in long-term DFS.

In conclusion, our study revealed that the early response to NACT was a predictor of DFS using data from a retrospective study. This predictor was also validated in data from a prospective cohort study. This result may help doctors and patients to predict long-term survival. Further study should identify as many risk factors as possible and combine the risk factors together to give clinicians a more definite method for predicting long-term survival among patients with cervical cancer who are treated with NACT and surgery.

\section{Clinical response}

Responder vs Non-responder

Stage

IIA vs IB2

IIB vs IB2

Grade

$\mathrm{G} 2$ vs $\mathrm{G} 1$

G3 vs $\mathrm{G} 1$

Undetermined vs G1

Cell type

Squamous vs non-squamous

Parametrial infiltration

Positive vs negative

Lymph node metastasis

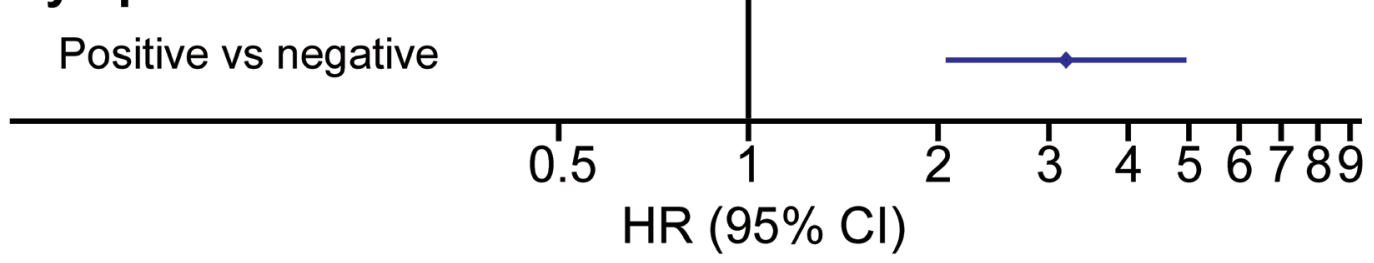

Figure 4: Univariate prognostic factor analysis in the prospective study. Univariate Cox analysis showed that several factors were significantly associated with DFS, including clinical response, age, FIGO stage, parametrial infiltration, vaginal surgical margin and lymph node metastasis. Abbreviations: HR, hazard ratio; FIGO, International Federation of Gynecology and Obstetrics; DFS, disease-free survival; LVSI, lymph vascular space invasion. 
Clinical response

Responder vs Non-responder

Age

$>44$ vs $\leq 44$ years

Vaginal surgical margin

Positive vs negative

Lymph node metastasis

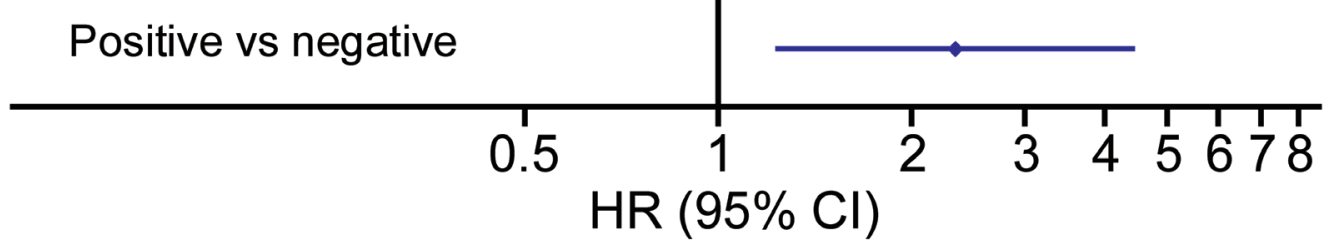

Figure 5: Multivariate prognostic factor analysis in the prospective study. Multivariate Cox analysis showed that several factors were significantly associated with DFS, including clinical response, age, vaginal surgical margin and lymph node metastasis. Abbreviations: HR, hazard ratio; FIGO, International Federation of Gynecology and Obstetrics; DFS, disease-free survival; LVSI, lymph vascular space invasion.

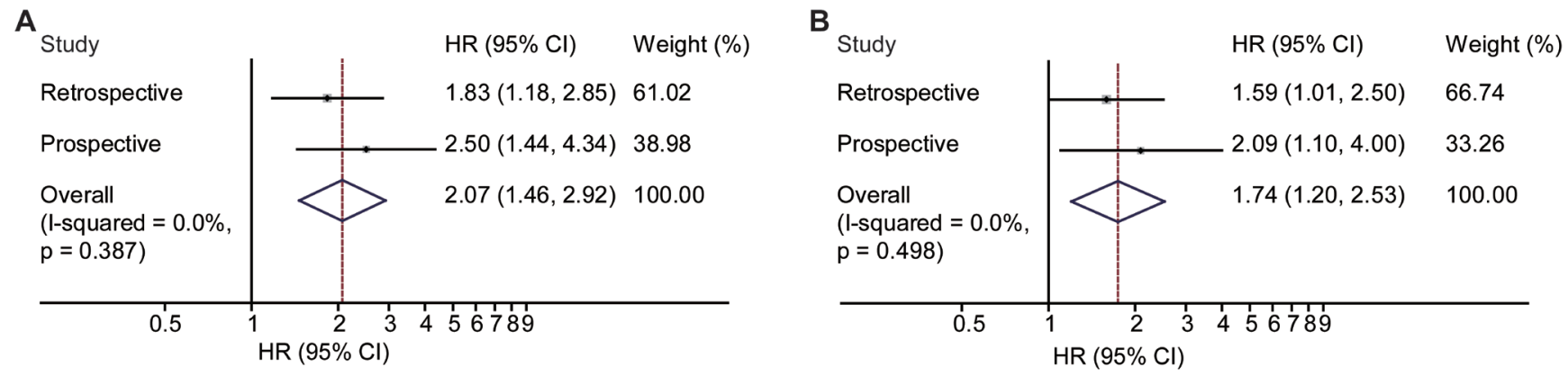

Figure 6: The combined clinical response results in both of the studies. Combined Cox analysis showed that the clinical response was significantly associated with DFS.A. meta-analysis for univariate Cox analysis; B. meta-analysis for multivariate Cox analysis.
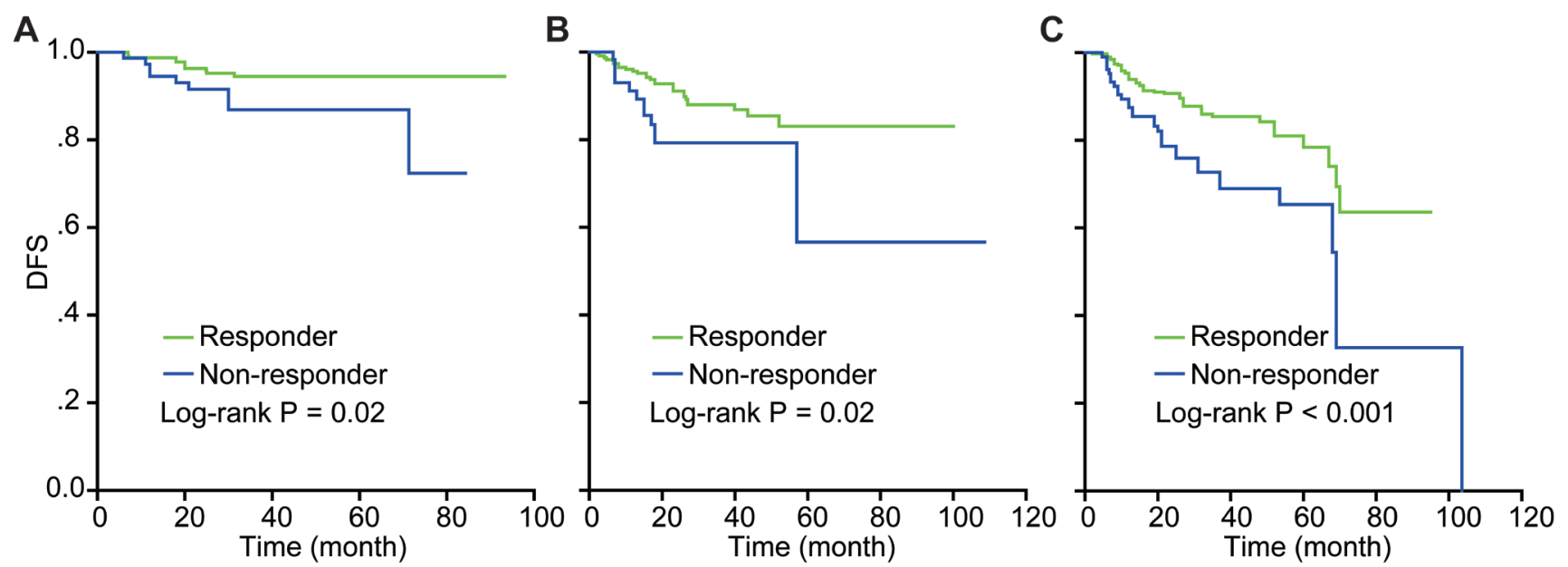

Figure 7: The DFS difference in each FIGO stage according to clinical response. A log-rank test showed that DFS in the responder group was significantly higher than in the non-responder group: A. $P=0.02$ for FIGO IB2; B. $P=0.02$ for FIGO IIA; C. $P<$ 0.001 for FIGO IIB. $P<0.05$ indicates statistical significance. 

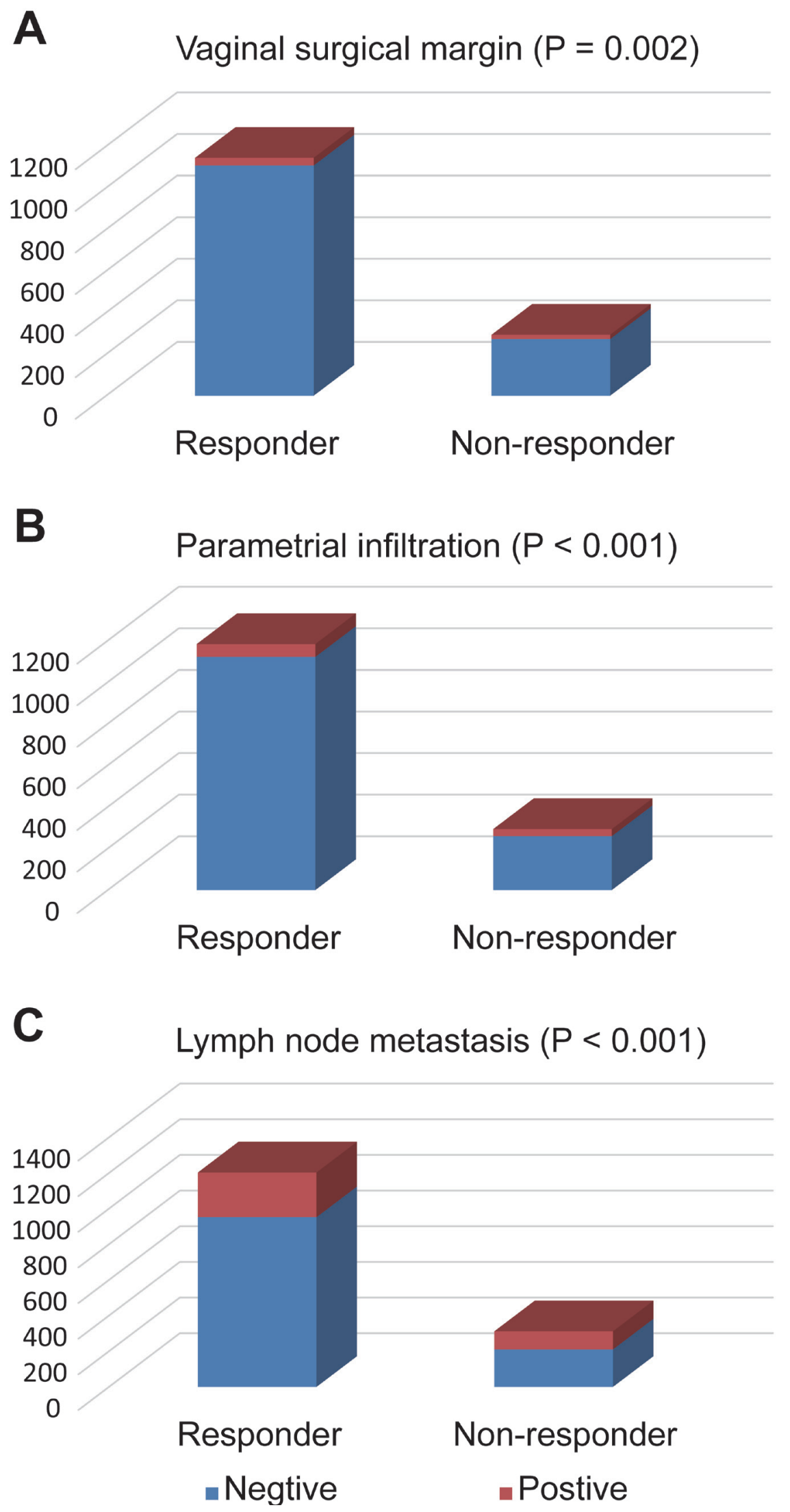

Figure 8: High-risk prognostic factors' distribution among the responders and non-responders. The ratio of cases with A. positive vaginal surgical margin $(P=0.002)$, B. positive parametrial infiltration $(P<0.001)$ or $\mathbf{C}$. positive lymph node $(P<0.001)$ were significantly high in the non-responder group: A. $P=0.002$; B. $P<0.001 ;$ C. $P<0.001$. 


\section{MATERIALS AND METHODS}

The information used in this study included data from a retrospective case-control study and a prospective cohort study (the registration number at Clinicaltrial.gov was NCT01628757). This study follows the Declaration of Helsinki and was carried out in accordance with the approved guidelines. All experimental protocols were approved by the ethical committee at Huazhong University of Science and Technology. All eligible patients gave written informed consent before entering this study.

\section{Eligibility criteria}

The patients' inclusion criteria were as follows: patients with cervical cancer diagnosed by pathologists and clinicians; age $\geq 18$ years old and $\leq 70$ years old; Karnofsky score $>70$; normal cardiac function; normal EKG; normal chest X-ray; normal hepatic function with normal total bilirubin $(\leq 1.5 \times$ the upper normal limit); normal renal function with normal serum creatinine level ( $\leq 1.5 \times$ the upper normal limit); WBC count $\geq 4 \times 10^{9} / \mathrm{L}$ and $\leq 10 \times 10^{9} / \mathrm{L}$; absolute neutrophil count $(\mathrm{ANC}) \geq 2$ $\times 10^{9} / \mathrm{L} ; \mathrm{HB}$ concentration $\geq 90 \mathrm{~g} / \mathrm{L} ;$ PLT count $\geq 100 \times$ $10^{9} / \mathrm{L}$

The patients were excluded for any of the following reasons: previously treated cervical cancer; history of other malignant tumor diseases; sepsis or other active infection; heart disease such as heart failure or myocardial infarction; withdrawing consent or leaving the study.

\section{Criteria to evaluate the clinical response}

The criteria adopted in this study are the WHO standards, which were also adopted by the previous studies $[19,39,40]$. Responders were defined as CR (complete response) + PR (partial response). CR means the disappearance of all tumor cells; PR means a resident tumor size less than $50 \%$ of the primary tumor size, without new lesions; SD (stable disease) means the tumor size reduction is less than $50 \%$ of the primary size, without new lesions; PD (progressive disease) means chemotherapy is useless, and the tumor size increases by greater than $25 \%$ or new lesions appear even after chemotherapy.

Ultrasound of the tumor as well as the pelvic condition was scheduled and examined after each NACT cycle to control for progressive disease among all patients. If the tumors were considered to be operable, radical surgery was performed within 4 weeks after the completion of the last scheduled chemotherapy cycle. Otherwise, the patients underwent CCRT. After the completion of the safety follow-up (i.e., 4 weeks after surgery), the decision regarding systemic adjuvant postsurgery therapy was at the discretion of the treating gynecologists. Patients who had risk prognostic factors, such as parametrial infiltration, positive lymph nodes, deep stromal invasion, lymph vascular involvement, or positive surgical margin, received postoperative irradiation or postoperative chemotherapy.

\section{Patient follow-up}

Patients were followed every three months in the first year, every six months in the second and third year, and every 12 months thereafter. The examination included pelvic MRI or computerized tomography (CT), pelvic ultrasound, physical examination of the pelvis by the gynecologist, chest X-ray, EKG, blood RT, renal function, and hepatic function.

\section{Statistical analysis}

The Kaplan-Meier method was used to construct the survival curves for DFS. A log-rank test was used to compare the survival rate between the responders and the non-responders. A univariate Cox regression model was used to evaluate the role of all the variables, including clinical factors and pathological factors, as significant prognostic factors. A multivariate Cox regression model was used to identify the independent prognostic factors among both the clinical factors and the pathological factors. All statistical analysis was performed using the IBM SPSS 20.0 statistical software package.

\section{ACKNOWLEDGMENTS}

We thank Prof. Xing Hui, Dr. Ting Hu, Dr. Ru Yang, Dr. Zhilan Chen, Dr. Shaoshuai Wang, Dr. Fangxu Tang, Dr. Jin Zhou, scholars in Zhejiang University, scholars in Thailand, and scholars in Iwate Medical University for the support.

\section{CONFLICTS OF INTEREST}

None.

\section{GRANT SUPPORT}

This research was supported by the grant from International S\&T Cooperation Program of China (No.2013DFA31400), Program for New Century Excellent Talents in University (NO.NECT-12-0646), the Foundation of China (973 Program; No.2009CB521808) and by grants from the National Natural Science Foundation of China (No. 81501217; 81300460; 81402160; 81302267; $81370469 ; 81302264 ; 81201639 ; 81300453 ; 81072132$; $81372781 ; 81071663 ; 81370469 ; 81230038$; 81230052; 30973472; 81001151; 81071663; 30973205; 30973184; 
81172464; 81101964) and National Major Science and Technology Project (No. 2009ZX09103-739).

\section{REFERENCES}

1. Torre LA, Bray F, Siegel RL, Ferlay J, Lortet-Tieulent J and Jemal A. Global cancer statistics, 2012. CA Cancer J Clin. 2015; 65(2):87-108.

2. Di J, Rutherford S, Wu J, Song B, Ma L, Chen J and Chu C. Knowledge of Cervical Cancer Screening among Women across Different Socio-Economic Regions of China. PLoS One. 2015; 10(12):e0144819.

3. IARC W. GLOBOCAN 2012: Estimated Cancer Incidence, Mortality and Prevalence Worldwide in 2012. 2014. Available: http://globocan.iarc.fr/Pages/fact_sheets_cancer. aspx? cancer $=$ cervix. Accessed 2014 Sep 5.

4. Zhang L, Huang H, Hou T, Wu S, Huang Q, Song L and Liu J. URG4 overexpression is correlated with cervical cancer progression and poor prognosis in patients with early-stage cervical cancer. BMC cancer. 2014; 14:885.

5. Ren Y, Li Y and Liu J. A modified shortened administration schedule for neoadjuvant chemotherapy with irinotecan and cisplatin in locally advanced cervical cancer. Int J Gynecol Cancer. 2011; 21(4):685-689.

6. Sardi JE, Giaroli A, Sananes C, Ferreira M, Soderini A, Bermudez A, Snaidas L, Vighi S, Gomez Rueda N and di Paola G. Long-term follow-up of the first randomized trial using neoadjuvant chemotherapy in stage Ib squamous carcinoma of the cervix: the final results. Gynecol Oncol. 1997; 67(1):61-69.

7. Sardi JE, Boixadera MA and Sardi JJ. Neoadjuvant chemotherapy in cervical cancer: a new trend. Curr Opin Obstet Gynecol. 2005; 17(1):43-47.

8. Linghu H, Xu XR, Mei YY, Tang JY, Tang LD and Sun T. Response of early stage bulky cervical squamous carcinoma to preoperative adjuvant chemotherapy. Chin Med Sci J. 2004; 19(2):116-119.

9. Huang X, Lan C, Huang H, Zhang Y, Cao X, Huang Y, Guo Y, Wan T and Liu J. Neoadjuvant docetaxel combined with cisplatin and followed by radical surgery for the treatment of locally advanced (stage IB2 - IIB) cervical cancer: preliminary results of a single-institution experience. Expert Opin Pharmacother. 2011; 12(2):165-173.

10. Sardi JE. Neoadjuvant chemotherapy in gynecologic oncology. Surg Clin North Am. 2001; 81(4):965-985.

11. Morice P, Uzan C, Gouy S, Verschraegen C and HaieMeder C. Gynaecological cancers in pregnancy. Lancet. 2012; 379(9815):558-569.

12. Rob L, Skapa P and Robova H. Fertility-sparing surgery in patients with cervical cancer. Lancet Oncol. 2011; 12(2):192-200.

13. Benedetti-Panici P, Greggi S, Colombo A, Amoroso M, Smaniotto D, Giannarelli D, Amunni G, Raspagliesi F, Zola $\mathrm{P}$, Mangioni $\mathrm{C}$ and Landoni F. Neoadjuvant chemotherapy and radical surgery versus exclusive radiotherapy in locally advanced squamous cell cervical cancer: results from the Italian multicenter randomized study. J Clin Oncol. 2002; 20(1):179-188.

14. Neoadjuvant chemotherapy for locally advanced cervical cancer: a systematic review and meta-analysis of individual patient data from 21 randomised trials. Eur J Cancer. 2003; 39(17):2470-2486.

15. Chen CA, Cheng WF, Wei LH, Su YN and Hsieh CY. Radical hysterectomy alone or combined with neoadjuvant chemotherapy in the treatment of early stage bulky cervical carcinoma. J Formos Med Assoc. 2002; 101(3):195-202.

16. Liu SP, Yang JX, Cao DY, Shen K, Xiang Y and Lang JH. Efficacy of neoadjuvant cisplatin and 5-flourouracil prior to surgery in FIGO stage IB2/IIA2 cervical cancer. Mol Clin Oncol. 2014; 2(2):240-244.

17. Choi CH, Song SY, Choi JJ, Park YA, Kang H, Kim TJ, Lee JW, Kim BG, Lee JH and Bae DS. Prognostic significance of VEGF expression in patients with bulky cervical carcinoma undergoing neoadjuvant chemotherapy. BMC cancer. 2008; 8:295.

18. Shoji T, Takatori E, Saito T, Omi H, Kagabu M, Miura F, Takeuchi S and Sugiyama T. Neoadjuvant chemotherapy using platinum- and taxane-based regimens for bulky stage Ib2 to IIb non-squamous cell carcinoma of the uterine cervix. Cancer Chemother Pharmacol. 2013; 71(3):657-662.

19. Hu T, Li S, Chen Y, Shen J, Li X, Huang K, Yang R, Wu L, Chen Z, Jia Y, Wang S, Cheng X, Han X, Lin Z, Xing $\mathrm{H}, \mathrm{Qu} \mathrm{P}$, et al. Matched-case comparison of neoadjuvant chemotherapy in patients with FIGO stage IB1-IIB cervical cancer to establish selection criteria. Eur J Cancer. 2012; 48(15):2353-2360.

20. Tierney JF, Stewart LA, Ghersi D, Burdett S and Sydes MR. Practical methods for incorporating summary time-toevent data into meta-analysis. Trials. 2007; 8:16.

21. Xiong Y, Liang LZ, Cao LP, Min Z and Liu JH. Clinical effects of irinotecan hydrochloride in combination with cisplatin as neoadjuvant chemotherapy in locally advanced cervical cancer. Gynecol Oncol. 2011; 123(1):99-104.

22. Li R, Lu ST, Si JG, Liu B, Wang H, Mei YY and Linghu H. Prognostic value of responsiveness of neoadjuvant chemotherapy before surgery for patients with stage $\mathrm{IB}(2) /$ IIA(2) cervical cancer. Gynecol Oncol. 2013; 128(3):524529.

23. Chen $\mathrm{H}$, Liang $\mathrm{C}$, Zhang $\mathrm{L}$, Huang $\mathrm{S}$ and $\mathrm{Wu} \mathrm{X}$. Clinical efficacy of modified preoperative neoadjuvant chemotherapy in the treatment of locally advanced (stage IB2 to IIB) cervical cancer: randomized study. Gynecol Oncol. 2008; 110(3):308-315.

24. Ye Q, Yuan HX and Chen HL. Responsiveness of neoadjuvant chemotherapy before surgery predicts favorable prognosis for cervical cancer patients: a metaanalysis. J Cancer Res Clin Oncol. 2013; 139(11):18871898. 
25. Biewenga P, van der Velden J, Mol BW, Stalpers LJ, Schilthuis MS, van der Steeg JW, Burger MP and Buist MR. Prognostic model for survival in patients with early stage cervical cancer. Cancer. 2011; 117(4):768-776.

26. Sevin BU, Lu Y, Bloch DA, Nadji M, Koechli OR and Averette HE. Surgically defined prognostic parameters in patients with early cervical carcinoma. A multivariate survival tree analysis. Cancer. 1996; 78(7):1438-1446.

27. Kamura T, Tsukamoto N, Tsuruchi N, Saito T, Matsuyama T, Akazawa K and Nakano H. Multivariate analysis of the histopathologic prognostic factors of cervical cancer in patients undergoing radical hysterectomy. Cancer. 1992; 69(1):181-186.

28. Lai CH, Chang CJ, Huang HJ, Hsueh S, Chao A, Yang JE, Lin CT, Huang SL, Hong JH, Chou HH, Wu TI, Huang KG, Wang CC and Chang TC. Role of human papillomavirus genotype in prognosis of early-stage cervical cancer undergoing primary surgery. J Clin Oncol. 2007; 25(24):3628-3634.

29. Ho CM, Chien TY, Huang SH, Wu CJ, Shih BY and Chang SC. Multivariate analysis of the prognostic factors and outcomes in early cervical cancer patients undergoing radical hysterectomy. Gynecol Oncol. 2004; 93(2):458-464.

30. Alvarez RD, Soong SJ, Kinney WK, Reid GC, Schray MF, Podratz KC, Morley GW and Shingleton HM. Identification of prognostic factors and risk groups in patients found to have nodal metastasis at the time of radical hysterectomy for early-stage squamous carcinoma of the cervix. Gynecol Oncol. 1989; 35(2):130-135.

31. Yuan CC, Wang PH, Lai CR, Yen MS, Chen CY and Juang CM. Prognosis-predicting system based on factors related to survival of cervical carcinoma. Int J Gynaecol Obstet. 1998; 63(2):163-167.

32. Deng T, Feng Y, Zheng J, Huang Q and Liu J. Low initial human papillomavirus viral load may indicate worse prognosis in patients with cervical carcinoma treated with surgery. J Gynecol Oncol. 2015; 26(2):111-117.

33. Giaroli A, Sananes C, Sardi JE, Maya AG, Bastardas ML, Snaidas L, Rueda NG, Vighi S and di Paola GR. Lymph node metastases in carcinoma of the cervix uteri: response to neoadjuvant chemotherapy and its impact on survival. Gynecol Oncol. 1990; 39(1):34-39.
34. Park JY, Kim DY, Kim JH, Kim YM, Kim YT and Nam JH. Further stratification of risk groups in patients with lymph node metastasis after radical hysterectomy for earlystage cervical cancer. Gynecol Oncol. 2010; 117(1):53-58.

35. Hu T, Li X, Zhang Q, Huang K, Jia Y, Yang R, Tang F, Tian $\mathrm{Q}, \mathrm{Ma} \mathrm{D}$ and Li S. Could the extent of lymphadenectomy be modified by neoadjuvant chemotherapy in cervical cancer? A large-scale retrospective study. PLoS One. 2015; 10(4): 0123539.

36. Suprasert P, Srisomboon J, Charoenkwan K, Siriaree S, Cheewakriangkrai C, Kietpeerakool C, Phongnarisorn $\mathrm{C}$ and Sae-Teng J. Twelve years experience with radical hysterectomy and pelvic lymphadenectomy in early stage cervical cancer. J Obstet Gynaecol. 2010; 30(3):294-298.

37. Sardi J, Sananes C, Giaroli A, Bayo J, Rueda NG, Vighi S, Guardado N, Paniceres G, Snaidas L, Vico C and et al. Results of a prospective randomized trial with neoadjuvant chemotherapy in stage IB, bulky, squamous carcinoma of the cervix. Gynecol Oncol. 1993; 49(2):156-165.

38. Buda A, Lissoni AA, Floriani I, Biagioli E, Gerardi C, Bonazzi C, Chiari S, Locatelli L, Dell'Anna T, Signorelli M, Mangioni C and Milani R. Long-Term Clinical Benefits of Neoadjuvant Chemotherapy in Women With Locally Advanced Cervical Cancer: Validity of Pathological Response as Surrogate Endpoint of Survival. Int J Gynecol Cancer. 2015.

39. Buda A, Fossati R, Colombo N, Fei F, Floriani I, Gueli Alletti D, Katsaros D, Landoni F, Lissoni A, Malzoni C, Sartori E, Scollo P, Torri V, Zola P and Mangioni C. Randomized trial of neoadjuvant chemotherapy comparing paclitaxel, ifosfamide, and cisplatin with ifosfamide and cisplatin followed by radical surgery in patients with locally advanced squamous cell cervical carcinoma: the SNAP01 (Studio Neo-Adjuvante Portio) Italian Collaborative Study. J Clin Oncol. 2005; 23(18):4137-4145.

40. Huang K, Li X, Yang R, Shen J, Chen Z, Qin X, Wang S, Jia Y, Tang F, Zhou H, Sun H, Zhou J, Guo L, Wang L, Qiao L, Xiong J, et al. Prospective cohort study to evaluate the efficacy of taxane plus platinum and CPT-11plus platinum regimes and to identify prognostic risk factors in cervical cancer patients. Int J Clin Exp Med. 2015; 8(9):15018-15029. 